\title{
Dosis acumulada en tomografía computada 2014-2017: análisis descriptivo de una población del Hospital de Clínicas
}

\author{
Liliana Servente Luquetti* ${ }^{*}$ Flavia Martinucci Silva ${ }^{\dagger}$, Nahiara Chozza Lecuna ${ }^{\dagger}$, Héctor \\ Hartmann Larronda†, María Irazoqui Cortazzo ${ }^{\dagger}$, Carolina Maguna Baubeta ${ }^{\dagger}$, Lucía Romero $^{\dagger}$ \\ Larrosa $^{\dagger}$, Luis Dibarboure Bassagoda ${ }^{\ddagger}$
}

\section{Resumen}

Introducción: el uso creciente de la tomografía computada produce un aumento de la dosis colectiva con el potencial riesgo de las radiaciones ionizantes. El objetivo del trabajo fue determinar la frecuencia de estudios tomográficos repetidos y estimar la dosis de radiación acumulada en el período 2014/2017 de una muestra de pacientes asistida durante julio/agosto de 2017 en el Hospital de Clínicas.

Método: se incluyeron pacientes que en julio/agosto de 2017 concurrieron al Departamento de Imagenología del Hospital de Clínicas, a quienes su médico tratante indicó la realización de una tomografía computada. Se trató de un estudio descriptivo, longitudinal, retrospectivo. Se completó una planilla con datos demográficos y del procedimiento. Se realizó la búsqueda de estudios tomográficos previos en el sistema de archivo de imágenes médicas y registro de informes de los últimos tres años.

Resultados: en la muestra se incluyeron 110 pacientes; $59(54,1 \%)$ se hicieron más de una tomografía, siendo abdomen y pelvis las regiones más estudiadas. Siete pacientes (6,4\%) superaron el umbral de dosis de $100 \mathrm{mSv}$ y $16(14,5 \%)$ recibieron dosis entre 50 y 100 mSv. A su vez, 23 pacientes (20,9\%) se hicieron más de cuatro tomografías, es decir, en promedio más de una al año.

Conclusiones: la tomografía computada es una herramienta beneficiosa para el diagnóstico siempre y cuando se use racionalmente haciendo un balance entre riesgo/beneficio. Se debe tomar conciencia de los riesgos vinculados a la dosis acumulada en múltiples estudios tomográficos.

Palabras clave: Tomografía computarizada por rayos $X$ Radiación ionizante

Protección radiológica

Dosis de radiación

Diagnóstico por imagen
Key words: $\quad$ Tomography, X-ray computed

Radiation, ionizing

Radiation protection

Radiation dosage

Diagnostic imaging

\footnotetext{
* Prof. Agdo. Departamento Clínico de Imagenología. Hospital de Clínicas Dr. Manuel Quintela. Montevideo, Uruguay.

† Bres. estudiantes del Ciclo de Metodología Científica II-2017.

‡ Prof. Departamento Clínico de Imagenología. Hospital de Clínicas Dr. Manuel Quintela. Montevideo, Uruguay.

Correspondencia: Liliana Servente, Av. Luis P. Ponce 1369/1002. Correo electrónico: Iservente@gmail.com

No existe apoyo financiero.

Los autores declaran que no existe conflicto de intereses.

Recibido: 26/2/18

Aprobado: 18/6/18
} 


\section{Introducción y antecedentes}

Tipos y fuentes de radiación: la población está expuesta a diario a radiación de origen natural y artificial. La dosis promedio de radiación natural es de $2,2 \mathrm{mSv} / \mathrm{anno}^{(1)}$.

Dentro de los estudios diagnósticos, los radiológicos y en especial la tomografía computada (TC) constituyen la fuente que más aporta a la dosis colectiva ${ }^{(2)}$. El uso médico de la radiación representa el $98 \%$ de la dosis poblacional con origen en fuentes artificiales y el $20 \%$ de la exposición total de la población ${ }^{(3)}$. En la figura 1 se muestra la evolución temporal de la contribución a la dosis efectiva de fuentes artificiales ${ }^{(4)}$.

Numerosos trabajos han demostrado el gran incremento de los estudios radiológicos diagnósticos en países desarrollados, en particular la TC (63 millones de estudios anuales en Estados Unidos), que representa el $60 \%$ de la radiación artificial, debiéndose este hecho a múltiples causas: aumento de la disponibilidad de tomógrafos, demanda de estudios por los propios pacientes, medicina defensiva, repetición de estudios por falta de información, entre otros ${ }^{(5-8)}$ (figura 2).

A pesar de las crecientes preocupaciones con respecto a la exposición a la radiación médica, todavía hay una conciencia limitada acerca de los riesgos de cáncer inducido $^{(9)}$. Una encuesta realizada en Estados Unidos entre los médicos de emergencia e imagenólogos encontró que el $75 \%$ de los mismos subestimó los riesgos de la radiación por la TC. El $53 \%$ de los radiólogos y $91 \%$ de los médicos de emergencia no creían que la TC pudiera aumentar el riesgo de cáncer ${ }^{(10)}$.

Magnitudes dosimétricas: la dosis de radiación absorbida por los tejidos se mide en Gray ${ }^{(11-13)}$. La dosis equivalente toma en consideración el tipo de radiación y la sensibilidad de los órganos y tejidos y se mide en Sie- vert $(\mathrm{Sv})$. Una radiografía de tórax $(\mathrm{Rx} T \mathrm{x})$ proporciona una dosis equivalente de $0,1 \mathrm{mSv}$. A menudo se utiliza una comparación con la dosis de la RxTx, de esta manera, por ejemplo, la dosis de una TC de abdomen corresponde en promedio a 75 radiografías de tórax ${ }^{(13-15)}$.

Efectos biológicos de las radiaciones ionizantes (RI): el uso de las RI produce diversos efectos en los tejidos biológicos.

Los efectos determinísticos, que son de tipo "todo o nada", tienen umbral de dosis y la severidad depende de la dosis recibida, pudiendo ocurrir quemaduras, cataratas y alteraciones en gónadas. Los efectos estocásticos no requieren umbral de dosis y son acumulativos. La probabilidad de aparición de estos efectos aumenta con la dosis y comprenden la aparición de cáncer y alteraciones genéticas.

Los diferentes tejidos biológicos tienen diferente sensibilidad a las RI, los más radiosensibles son las gónadas, cristalino, tiroides y mama ${ }^{(16)}$. La radiosensibilidad de los tejidos depende también de la edad y el sexo; en líneas generales, las personas más vulnerables son las mujeres y los niños (debido a su menor espesor corporal, mayor porcentaje de células en mitosis, y una expectativa de vida más larga ${ }^{(16)}$. Los efectos de la exposición en el embarazo dependen de la edad gestacional y de la dosis absorbida ${ }^{(17)}$.

El riesgo de desarrollar cáncer con radiaciones a bajas dosis se ha estudiado en base a sobrevivientes de las bombas atómicas de Hiroshima y Nagasaki y en trabajadores que sufrieron accidentes con materiales radiacti$\operatorname{vos}^{(18,19)}$. Los estudios epidemiológicos realizados en poblaciones expuestas a la radiación han mostrado un aumento significativo del riesgo de cáncer con dosis superiores a $100 \mathrm{mSv}^{(19-23)}$.

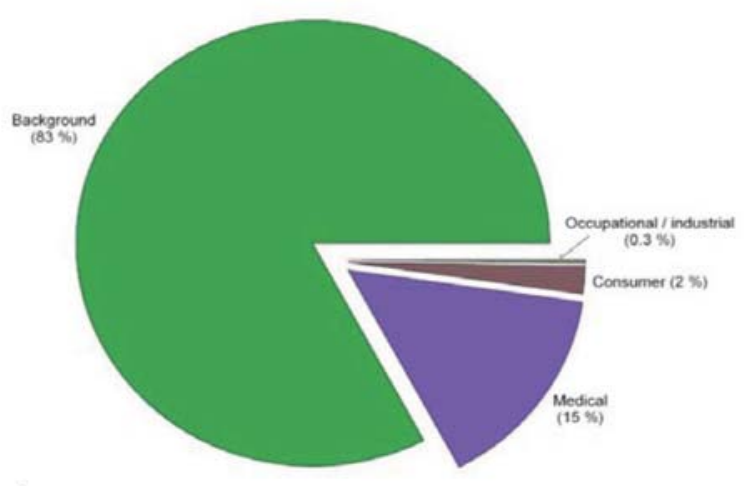

a.

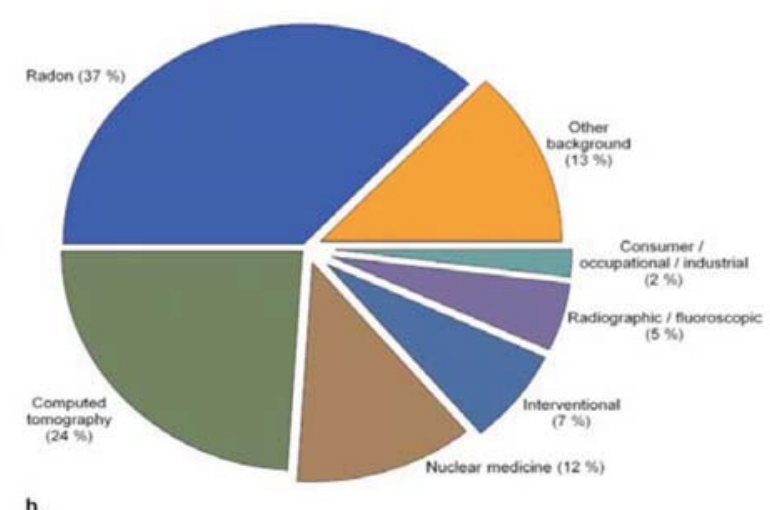

b.

Figura 1. Promedio de la dosis efectiva de las principales fuentes de exposición: a) Dosis efectivas a principios de la década de 1980. b) Dosis efectivas en $2006^{(4)}$. 


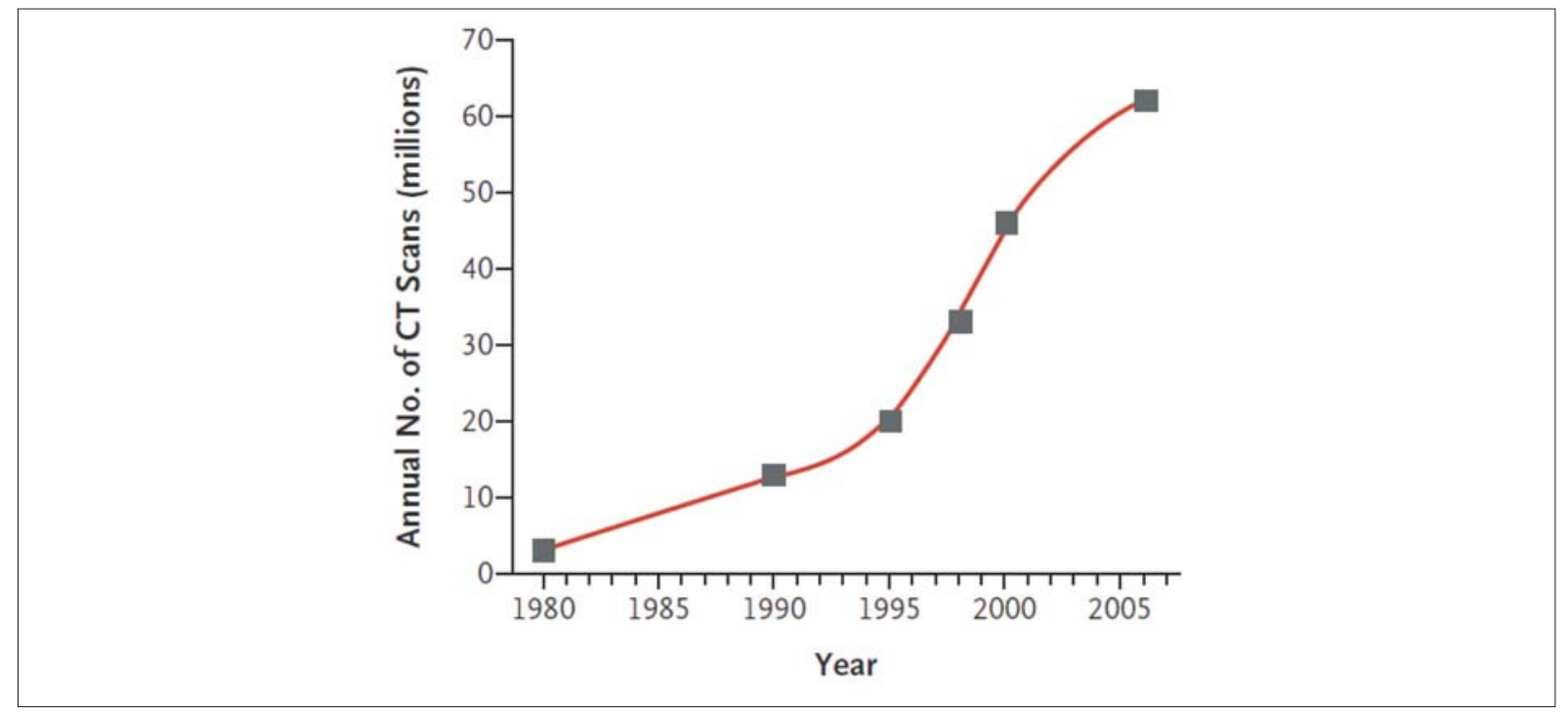

Figura 2. Número de tomografías computadas estimadas anualmente en Estados Unidos ${ }^{(5)}$.

Pearce y colaboradores ${ }^{(24)}$ realizaron un estudio retrospectivo entre 1985 y 2002 para evaluar si el riesgo de leucemia y tumores encefálicos se incrementó después de realizadas TC en pacientes menores de 22 años sin diagnóstico previo de cáncer. Se comprobó que dosis acumuladas de $50 \mathrm{mSv}$ triplicaban el riesgo de leucemia y dosis de $60 \mathrm{mSv}$ triplicaban el riesgo de tumor cerebral.

Los efectos biológicos de las radiaciones ionizantes a baja dosis se resumen en el reporte realizado por la Academia Nacional de Ciencia de Estados Unidos que actualmente se encuentra en su edición VII, Biological effects of ionizing radiation (BEIR VII) ${ }^{(25)}$. Dicho modelo asume una relación lineal, sin umbral, es decir que la probabilidad de ocurrencia de un cáncer radioinducido aumenta proporcionalmente con la dosis recibida. Plantea que el riesgo es inversamente proporcional a la edad, cae luego de los 40 años y es mayor en mujeres. Este modelo ha sido ampliamente debatido; sin embargo, hasta el momento se lo considera el más adecuado.

Tomografía computada en Uruguay: de acuerdo a datos proporcionados por la División de Tecnología del Ministerio de Salud Pública y a través de encuestas realizadas a médicos imagenólogos, hasta diciembre de 2016 existían en Uruguay 49 equipos de TC distribuidos en todos los departamentos. Para dicho relevamiento no se tuvo en cuenta: TC de uso exclusivo para radioterapia, ni los sistemas híbridos SPECT o PET/TC. En la figura 3 se muestra la evolución temporal del número de tomógrafos en Uruguay, en la misma se observa un incremento lineal hasta el año 2015, en el que se alcanza una meseta.
La medición de la dosis de RI recibida en TC se realiza en base al reporte de dosis: CTDIvol (índice de dosis volumétrico TC) y DLP (producto dosis-longitud). A partir de estos parámetros y según las regiones a estudiar, se llega al cálculo de dosis equivalente. Estas medidas son aproximadas y estimativas de la dosimetría real que recibe el paciente ${ }^{(26)}$.

El Departamento Clínico de Imagenología del Hospital de Clínicas cuenta con un tomógrafo multicorte de 64 hileras. Según datos proporcionados por el archivo de radiología, disponible en la página web del Hospital de Clínicas ${ }^{(27)}$, en el año 2016 se realizaron un total de 12.480 $\mathrm{TC}$, lo que supone un promedio de 1.040 TC mensuales.

A modo de resumen: el uso creciente de la TC contribuye cada vez más a la dosis colectiva. La probabilidad de efectos estocásticos se incrementa con la dosis, es acumulativa y hay evidencia que plantea un aumento en el desarrollo de cáncer radioinducido. Por lo tanto, los estudios que se basan en RI deben estar justificados, y en caso de ser realizados, hacer su optimización utilizando la menor dosis razonablemente posible (principio ALARA).

Objetivo: determinar la frecuencia de estudios tomográficos repetidos y estimar la dosis acumulada en el período 2014 a 2017 de una muestra de pacientes asistida en el Servicio de Tomografía del Hospital de Clínicas en los meses de julio y agosto de 2017.

\section{Material y método}

Diseño del estudio: se trató de un estudio de tipo descriptivo, observacional, longitudinal, retrospectivo.

Población: pacientes que concurrieron al Servicio de Tomografía del Hospital de Clínicas entre el 21 de ju- 


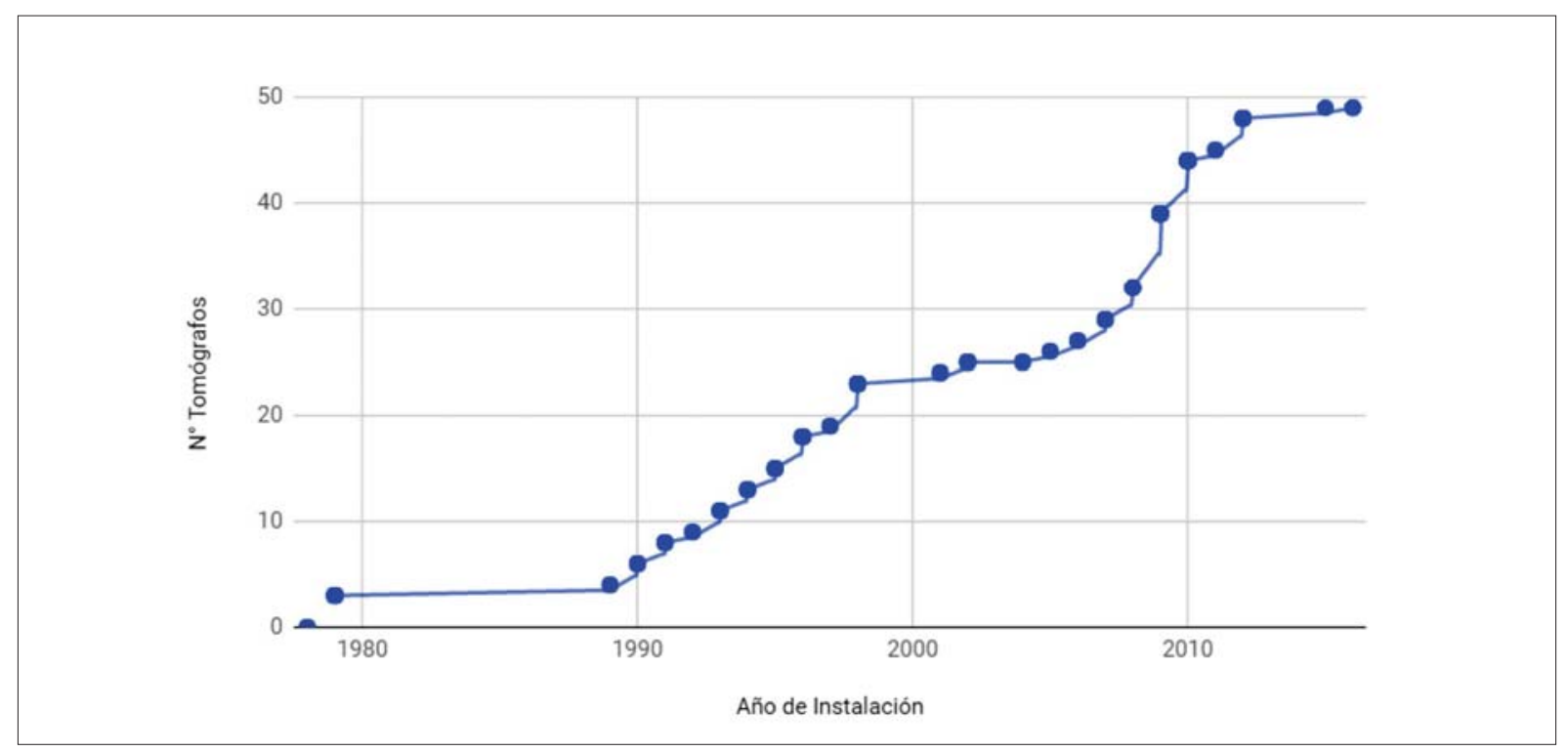

Figura 3. Evolución del número de tomógrafos en función del tiempo en Uruguay.

lio y el 15 de agosto del 2017 con indicación de TC. Los criterios de inclusión fueron los pacientes mayores de 18 años que dieron consentimiento informado. La muestra colectada es una muestra por conveniencia.

Variables: se relevaron las variables demográficas: sexo, edad, motivo de indicación de la TC, procedencia de la solicitud, región a estudiar, número de tomografías previas, dosis por estudio y dosis acumulada. El motivo de indicación de TC se categorizó en oncológico, no oncológico agudo y no oncológico crónico. La procedencia de la solicitud se categorizó en: policlínica ambulatoria, emergencia, centro de tratamiento intensivo (CTI) e internación general. La región a estudiar se clasificó en: cráneo, cuello/columna cervical, columna dorsal, columna lumbosacra, tórax, abdomen, pelvis y miembros. La dosis por estudio dependerá de la región a estudiar, según tablas de equivalencia (tabla 1). La dosis acumulada es la resultante de la suma de las dosis por estudio.

Procedimientos para recolección de la información: se realizó en forma retrospectiva a tres años la búsqueda de estudios previos de TC a partir de datos del PACS y del sistema de registro de informes del hospital (gestión asistencial). La información se ingresó a planillas diseñadas con tal fin. Se asignó un código para las variables discretas.

Aspectos éticos: el protocolo de investigación fue revisado y aprobado por el comité de ética del Hospital de Clínicas. Se garantizó la confidencialidad de los resultados, la anonimización de los datos y se entregó a los pacientes un formulario de consentimiento informado acerca del propósito de la investigación.
Análisis estadístico: para las variables cualitativas los resultados se expresaron en porcentajes y las variables cuantitativas se resumieron mediante medidas de tendencia central (media) y de dispersión (desvío estándar). Para el análisis de los datos se utilizó el software Epi Info versión 7.2.1.0.

\section{Resultados}

\section{Características de la muestra}

En el período del 21 de julio al 15 de agosto de 2017 un total de 110 pacientes pudieron ser incluidos en la investigación; 52 fueron mujeres $(47,3 \%)$ y 58 varones $(52,7 \%)$. La edad media fue 54,4 años (rango 18 a 88 ) con un desvío estándar de 17,5.

La procedencia de la solicitud fue $46,4 \%$ de policlínica ambulatoria; $28,2 \%$ de emergencia, y $25,5 \%$ internación general (figura 4a). El motivo de consulta fue: $44,5 \%$ patología no oncológica aguda; $40,9 \%$ patología oncológica; $14,5 \%$ patología no oncológica crónica (figura $4 b$ ).

\section{Características de la muestra con tomografías computadas repetidas}

Un total de 59 pacientes, que corresponden al $54,1 \%$ de la muestra, tuvieron más de una TC en los tres últimos años. De ellos, el 50,8\% fueron hombres y $49,1 \%$ mujeres. La edad media fue de 55,5 años (rango 19 a 81) con un desvío estándar de 16,2.

La procedencia de la solicitud fue $59,3 \%$ de policlínica ambulatoria; 20,3\% de emergencia; 20,3\% internación general. El motivo de consulta fue $55,9 \%$ patología 


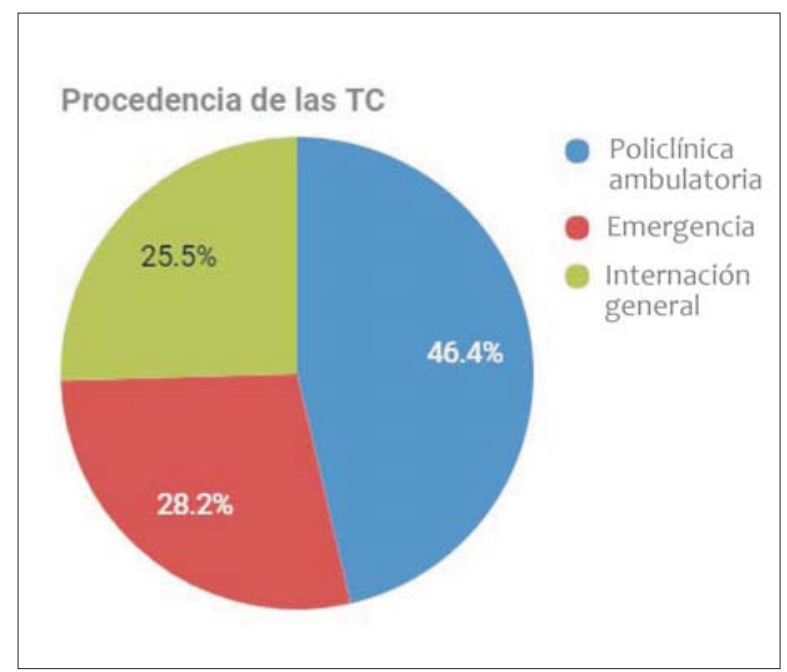

Figura 4a. Procedencia de solicitud de tomografía computada de la muestra atendida en los meses de julio y agosto de 2017 en el Departamento de Imagenología del Hospital de Clínicas.

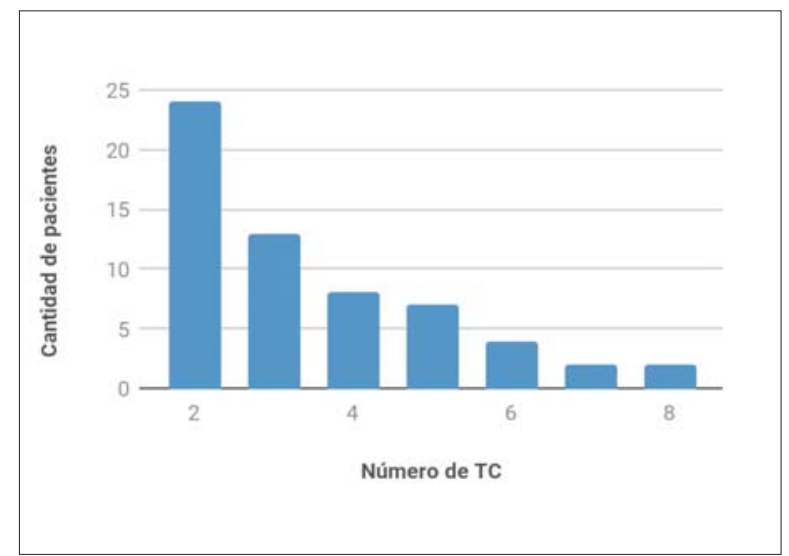

Figura 5. Número de tomografías computadas realizadas por pacientes del subgrupo con estudios tomográficos repetidos.

oncológica; $27,1 \%$ patología no oncológica aguda, y $16,9 \%$ no oncológica crónica.

La distribución de la localización anatómica para las TC correspondió en $64 \%$ de los casos a abdomen y pelvis, siendo la región más estudiada.

La dosis acumulada en la muestra en el período 2014-2017 tuvo una media de 50,3 mSv y un desvío estándar de $37,1 \mathrm{mSv}$, con un mínimo de 4 y un máximo de $184 \mathrm{mSv}$.

Destacamos que siete pacientes $(6,4 \%)$ superaron el umbral de los $100 \mathrm{mSv}$ y que 16 pacientes $(14,5 \%)$ recibieron dosis entre 50 y $100 \mathrm{mSv}$. A su vez, 23 pacientes $(20,9 \%)$ se hicieron más de cuatro tomografías, es decir, en promedio más de una TC anual.

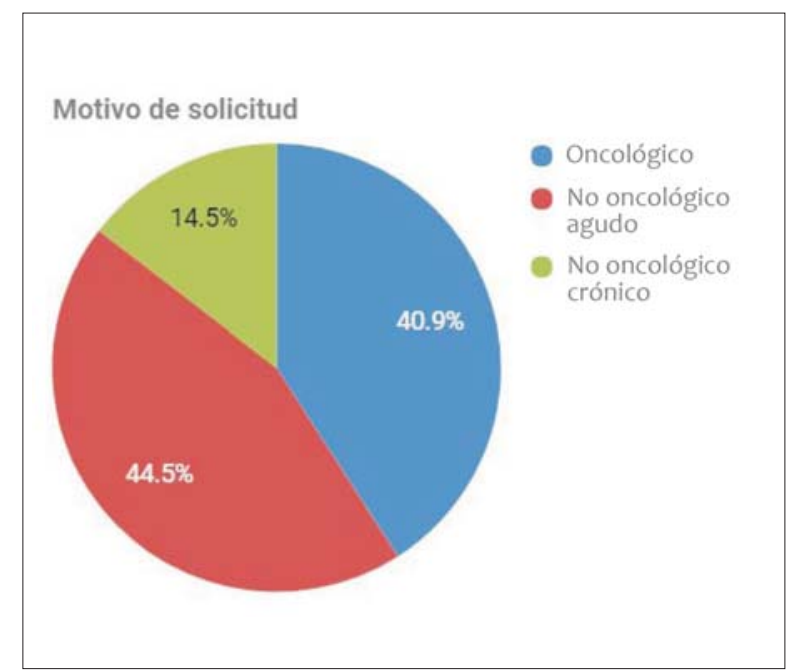

Figura $\mathbf{4 b}$. Motivo de solicitud de tomografía computada de la muestra.

La cantidad de TC que se realizaron en este grupo se muestra en la figura 5 .

En la tabla 2 se muestran los tres casos de la serie que tienen la mayor dosis acumulada.

\section{Discusión}

En el presente trabajo encontramos tasas de repetición de TC de $54,1 \%$, con el $20,9 \%$ de los pacientes sometidos a más de cuatro TC (más de una TC anual). El 6,4\% de la muestra acumuló dosis superiores a $100 \mathrm{mSv}$, rango en el que hay evidencia epidemiológica del aumento del riesgo de cáncer ${ }^{(16,28,29)}$.

El 64\% de las TC realizadas en la subpoblación de pacientes con estudios repetidos corresponden a abdomen y pelvis, lo que explicaría en parte un aumento en la dosis total acumulada, ya que la región de abdomen y pelvis es una de las que más contribuyen en cuanto a la dosis equivalente según la tabla 1.

En el trabajo realizado por Sodickson y colaboradores ${ }^{(15)}$ durante un período de 22 años y con una cohorte de 31.642 pacientes, el $15 \%$ de los mismos había acumulado una dosis superior a $100 \mathrm{mSv}$, con el $33 \%$ de los pacientes sometidos a más de cinco exámenes de TC y el $5 \%$ de los pacientes sometidos por lo menos a 22 exámenes. La conclusión de este trabajo es que aquellos pacientes que por su patología subyacente deben ser sometidos a controles con TC, tienen más riesgo de cáncer radioinducido.

Griffey y colaboradores ${ }^{(6)}$ estudiaron una población seleccionada de 130 pacientes que consultaron en el departamento de emergencia con al menos tres consultas anuales y con un período de seguimiento de 7,7 años. $\mathrm{La}$ mediana de TC fue de 10 , media 13 , con un máximo de 70. La dosis media fue de $122 \mathrm{mSv}$ y el máximo de 579 
$\mathrm{mSv}$. Un total de 55 pacientes superaron el umbral de dosis de $100 \mathrm{mSv}$. Los estudios de abdomen y pelvis representaron $53 \%$ del total.

En un estudio realizado en pacientes de $\mathrm{CTI}^{(14)}$, donde se llevó a cabo un relevamiento de las TC realizadas en 19.524 pacientes ingresados entre diciembre de 2011 y enero de 2017 para predecir su exposición a la radiación y calcular su riesgo estimado de cáncer (LAR), se encontró que la mayoría de las TC realizadas fueron: de cráneo, representando 49,9\% (35.403), mientras que abdomen-pelvis, tórax, columna y cuello representaron $22,7 \%$ (16.102), 16,8\% (11.912), $5,9 \%(4.200)$ y $4,7 \%(3.317)$, respectivamente. En nuestro caso, el $64 \%$ de las TC realizadas correspondieron a abdomen y pelvis.

Shah y colaboradores ${ }^{(8)}$, en el departamento de emergencia, buscaron determinar cuántos pacientes fueron sometidos a más de diez TC en un período de siete años (enero 2001-diciembre 2007). Para un total de 24.393 pacientes hubo 34.671 estudios tomográficos; 17.909 se hicieron una sola tomografía. Pero 26 pacientes $(0,1 \%)$ se realizaron más de diez TC con una dosis media de $83,4 \mathrm{mSv}$. Concluyeron que si bien los pacientes que tuvieron más de diez TC fueron una minoría, los mismos fueron sometidos a dosis que potencialmente pueden producir cáncer radioinducido.

Los resultados de estos trabajos no son estrictamente comparables con el nuestro, ya que se trata de poblaciones diferentes. Slovis y colaboradores estudian una población de CTI; Griffey y Shah estudian una población de emergencia. A su vez, los períodos de evaluación fueron diferentes.

Con respecto al trabajo de Sodickson y colaboradores, estos autores encontraron niveles de dosis de radiación y de repetición de estudios tomográficos mayores a los de nuestra investigación ( $15 \%$ y $6,4 \%$ que superaron el umbral de $100 \mathrm{mSv}$ respectivamente). Sin embargo, este autor estudió una cohorte durante 22 años. También debemos tener en cuenta que estos datos son de Estados Unidos, donde probablemente la población tenga más acceso a la TC y que en la práctica médica haya un mayor uso de la medicina defensiva.

El presente trabajo se llevó a cabo en un centro hospitalario universitario de tercer nivel de atención, de referencia a nivel nacional y de acceso público. Los pacientes presentan patologías complejas que quizá demanden más estudios imagenológicos. Todos estos factores, además de una medicina defensiva y una mayor demanda por parte del paciente, podrían influir en el número de solicitudes de TC.

Con respecto al motivo de solicitud de la TC, encontramos que en la muestra de 110 pacientes la patología no oncológica aguda fue la predominante $(44,5 \%)$,
Tabla 1. Estimación de la dosis efectiva en tomografía computada según la región anatómica; tomado y adaptado de referencia 15.

\begin{tabular}{lc}
\hline Región anatómica & Dosis efectiva TC (mSv) \\
\hline Cráneo/Cara & 2 \\
Columna cervical/Cuello & 2 \\
Columna dorsal/Tórax & 8 \\
Abdomen & 7,5 \\
Pelvis & 7,5 \\
Abdomen y pelvis/Columna lumbar & 15 \\
Extremidades & 0 \\
\hline
\end{tabular}

mientras que en el grupo de pacientes con TC repetidas el motivo oncológico fue el más frecuente $(55,9 \%)$. Esto es esperable pues el grupo que se controla más frecuentemente con TC es el de pacientes oncológicos.

La correcta justificación de los estudios es fundamental, realizar los estudios solo cuando son estrictamente necesarios teniendo en cuenta el riesgo/beneficio y la posibilidad de usar otros métodos de imagen que no utilicen RI (ecografía o resonancia magnética [RM]).

Para ello, se han desarrollado en muchos países guías de indicaciones apropiadas de los estudios de utilidad para el clínico; en la región, están disponibles en Argentina ${ }^{(30)}$.

Se han propuesto también niveles de referencia de dosis que se han elaborado realizando encuestas y considerando los CTDIvol y DLP de múltiples centros, se encuentran disponibles en diferentes países ${ }^{(31)}$. También es fundamental contar con equipamiento adecuado, entrenamiento del personal, optimización de protocolos, auditoría de dosis como parte del control de calidad. Debemos optimizar los estudios TC utilizando el principio ALARA: As Low As Reasonable Achievable, que implica utilizar el mínimo necesario de dosis para realizar un diagnóstico. Debemos adecuar la dosis de radiación al tamaño del paciente, ajustando parámetros técnicos (corriente y kilovoltaje del tubo, pitch), ajuste automático de exposición, limitar estudios a la región anatómica solicitada, realizar estudios de una sola fase y no multifase. Recientemente se han desarrollado softwares que utilizan algoritmos de reconstrucción iterativos que permiten utilizar menor dosis sin detrimento en la calidad de las imágenes. Para ello es importante la formación de un equipo multidisciplinario y la interrelación con el físico médico.

Para disminuir la repetición de estudios TC es fundamental, para el clínico solicitante, tener información de 
Tabla 2. Casos clínicos de los tres pacientes con mayor dosis acumulada; se resumen edad, sexo, procedencia de la solicitud, breve historia médica e imagenológica, dosis acumulada.

\begin{tabular}{|c|c|c|c|}
\hline Parámetros & Paciente 1 & Paciente 2 & Paciente 3 \\
\hline Edad/Sexo & 50 años/femenino & 50 años/masculino & 73 años/femenino \\
\hline Consulta & Policlínica de Oncología & Policlínica de Oncología & Policlínica de Oncología \\
\hline Historia médica & $\begin{array}{l}\text { Cáncer de colon estadio IV } \\
\text { pulmonar y hepático, diagnosticado } \\
\text { en } 2012 \\
\text { Controles TC para evaluar } \\
\text { respuesta postratamiento QT (Abr. } \\
\text { 2015) y RT (Set. 2015) }\end{array}$ & $\begin{array}{l}\text { Cáncer testicular, operado en } 2013 . \\
\text { Se realizan controles TC }\end{array}$ & $\begin{array}{l}\text { Cáncer de mama, diagnóstico } 2012 \\
\text { Se realizan controles TC }\end{array}$ \\
\hline Historia imagenológica & $\begin{array}{l}8 \text { TC de tórax, abdomen y pelvis } \\
\text { Nov. 2014, Abr. 2015, Set. 2015, } \\
\text { Nov. 2015, Feb. 2016, Jun. 2016, } \\
\text { Dic. 2016, Jul. } 2017\end{array}$ & $\begin{array}{l}6 \text { TC de tórax, abdomen y pelvis } \\
\text { Oct. 2014, Ene. 2015, Dic. 2015, } \\
\text { Jun. 2016, Ene. 2017, Ago. } 2017\end{array}$ & $\begin{array}{l}5 \text { TC de tórax, abdomen y pelvis: } \\
\text { Ago. 2014, Nov. 2015, Dic. 2015, } \\
\text { Abr. } 2016 \text { (suma cráneo), Ago. } \\
2017 . \\
1 \text { TC de columna cervical, dorsal y } \\
\text { lumbar, Ene. } 2015\end{array}$ \\
\hline Dosis acumulada & $184 \mathrm{mSv}$ & $138 \mathrm{mSv}$ & $124,5 \mathrm{mSv}$ \\
\hline Hallazgos en imágenes & $\begin{array}{l}\text { Nov. 2015, respuesta } \\
\text { imagenológica completa a } \\
\text { tratamiento con RT }\end{array}$ & $\begin{array}{l}\text { Oct. 2014, micronódulo pulmonar } \\
\text { sin cambios } \\
\text { Dic. 2015, crecimiento ganglionar a } \\
\text { nivel intercavo-aórtico } \\
\text { Nov. } 2017 \text {, aumento de las } \\
\text { adenopatías } \\
\text { Ago 2017, control pos-tratamiento, } \\
\text { regresión del conglomerado } \\
\text { ganglionar }\end{array}$ & $\begin{array}{l}\text { Ene. 2015, no se observa } \\
\text { secundarismo } \\
\text { Nov. 2015, lesiones a nivel óseo } \\
\text { Abr. 2016, incremento de } \\
\text { marcadores tumorales } \\
\text { Ago. 2017, evolución de lesiones a } \\
\text { nivel óseo }\end{array}$ \\
\hline
\end{tabular}

los antecedentes del paciente a través de herramientas como la historia clínica electrónica. Podría implementarse una planilla de registro de dosis individual para cada paciente, que sería de utilidad incluso para evitar errores por falta de información, por ejemplo la realización de un mismo estudio tomográfico solicitado en dos oportunidades diferentes por diferentes médicos en un corto lapso de tiempo. En la tabla 3 se propone un modelo de planilla de registro de dosis. Este historial dosimétrico individual fue propuesto por la normativa de la EURATOM en 2013 y será obligatorio en Europa para los estudios TC e intervencionistas desde el $2018^{(32)}$.

\section{Limitaciones}

Como limitaciones de este trabajo podemos tener en cuenta las siguientes consideraciones.

Estimamos una dosis acumulada mínima, pues no se tomó en cuenta la dosis real debida a varias adquisiciones (con contraste, arterial, portal, tardía), de todas maneras esta opción es válida, ya que otros autores también la proponen ${ }^{(8,15)}$.
Se utilizó una tabla de equivalencia de dosis según la región estudiada propuesta por algunos autores ${ }^{(15)}$; sin embargo, estos valores no necesariamente son trasladables al equipo de tomografía del Hospital de Clínicas. La falta de material adecuado y calibrado en el país para realizar las estimaciones dosimétricas correspondientes impidieron la estimación real de las dosis.

No se consideró el aporte de dosis debido a TC realizadas en otras instituciones en el período de tiempo estipulado.

Se consideró únicamente el período de 2014 a 2017, ya que previamente al año 2014 no existía un registro informático de los reportes y de los estudios de los pacientes atendidos en el departamento.

Se encontraron limitaciones en lo que respecta al registro adecuado de los pacientes.

La muestra de pacientes no fue representativa de la población del hospital por dificultades inherentes a la obtención del consentimiento informado. 
Tabla 3. Formato de planilla de registro de dosis individual propuesto.

\begin{tabular}{|c|c|c|c|c|}
\hline Nombre & & & & \\
\hline \multicolumn{5}{|l|}{$N^{\circ}$ de registro } \\
\hline Fecha & Tipo de estudio & Región irradiada & Dosis individual (mSv) & Dosis acumulada (mSv) \\
\hline & & & & \\
\hline & & & & \\
\hline & & & & \\
\hline
\end{tabular}

\section{Perspectivas}

Proponemos desarrollar e implementar un registro dosimétrico individual con el fin de documentar la dosis acumulada en RI tanto en los estudios de imagen como en los procedimientos terapéuticos. Elaborar guías o protocolos de solicitud adecuada de imágenes, principalmente en oncología.

Mayor período de recolección de datos reclutando un mayor número de pacientes estudiados, incluyendo a la población de CTI que en el presente trabajo no estuvo representada. Realizar estudios en poblaciones vulnerables, como lo es la pediátrica por su mayor radiosensibilidad.

\section{Conclusiones}

El uso generalizado de TC representa uno de los avances más importantes en el diagnóstico imagenológico. Sin embargo, comparado con la radiografía simple, la TC implica dosis de radiaciones más altas, resultando en un marcado incremento en la exposición a RI en la población sometida a este tipo de estudio.

El 6,4\% de los pacientes de nuestra muestra acumuló una dosis de radiación que sobrepasa los $100 \mathrm{mSv}$, rango en el que existe mayor riesgo de carcinogénesis inducida por radiación.

La TC es una herramienta útil en el ámbito médico siempre y cuando se use racionalmente, haciendo un balance entre riesgos y beneficios.

Proponemos la utilización de una planilla de registro de dosis individual para conocer la dosis acumulada por el paciente en estudios diagnósticos.

\section{Summary}

Introduction and objective: the rising use of computed tomography results in an increase in the collective doses with the potential risk for ionizing radiation. The study aimed at determining the frequency of repeated scans and at estimating the cumulative radiation doses between 2014 and 2017 in a sample of patients seen during July/August of 2017 at the Clinicas Hospital.

Method: patients who consulted at the Imagenology Department of the Clinicas Hospital in July and August, 2017 whose treating physician indicated a computed tomography were included in the study. It consisted of a descriptive, longitudinal, retrospective study. A spreadsheet with demographic data and procedure was filled out and previous tomographic studies were looked for in the medical images file system, and reports issued in the last 3 years were analysed.

Results: 110 patients made up the sample; more than one tomography was performed to $59(54.1 \%)$ patients underwent more than one scan, abdominal and pelvic scans being the most frequent. 7 patients $(6.4 \%)$ surpassed the $100 \mathrm{mSv}$ dose threshold and $16(14.5 \%)$ received doses between 50 and $100 \mathrm{mSv}$. Likewise, 23 patients $(20.9 \%)$ underwent more than 4 tomographies, that is more than one per year.

Conclusions: computed tomography is a beneficious diagnostic tool, provided it is used rationally upon a risk/benefit balance analysis. Awareness needs to be raised in relation to the risks associated to cumulative doses from multiple scans

\section{Resumo}

Introdução e objetivo: o uso crescente da tomografia computada produz um aumento da dose coletiva com o potencial risco das radiações ionizantes. O objetivo deste trabalho foi determinar a frequência de estudos tomográficos repetidos e estimar a dose de radiação acumulada no período 2014/2017 de uma amostra de pacientes atendida durante os meses de julho e agosto de 2017 no Hospital de Clínicas.

Método: foram incluídos pacientes que nos meses de julho e agosto de 2017 foram ao Depto. de Imagenología do HC com indicação de una tomografia computada por seu médico tratante. Realizou-se um estudo des- 
critivo, longitudinal e retrospectivo. Os dados foram coletados em uma planilha com dados demográficos e do procedimento. Fez-se uma pesquisa de estudos tomográficos prévios no sistema de arquivo de imagens médicas e registro de expedientes dos últimos 3 anos.

Resultados: cento e dez pacientes foram incluídos na amostra; 59 (54,1\%) fizeram mais de uma tomografia, sendo abdômen e pélvis as regiões mais estudadas. 7 pacientes $(6,4 \%)$ superaram o umbral de dose de 100 $\mathrm{mSv}$ e $16(14,5 \%)$ receberam doses entre 50 e $100 \mathrm{mSv}$. Por outro lado 23 pacientes $(20,9 \%)$ foram submetidos a mais de 4 tomografias, isto é, mais de uma por ano.

Conclusões: a tomografia computada é uma ferramenta útil para o diagnóstico sempre e quando seja usada racionalmente fazendo um balanço risco/beneficio. Deve-se tomar consciência dos riscos vinculados às doses acumuladas em múltiplos estudos tomográficos.

\section{Bibliografía}

1. Canadian Nuclear Safety Commission. Introduction to Radiation. Ottawa: CNSC, 2012. Disponible en:http://nuclearsafety.gc.ca/eng/pdfs/Reading-Room/radiation/Introduction-to-Radiation-eng.pdf [Consulta: octubre 2017].

2. Kalra MK, Sodickson AD, Mayo-Smith WW. CT radiation: key concepts for gentle and wise use. RadioGraphics 2015; 35(6):1706-21. Disponible en: http://pubs.rsna.org/ doi/10.1148/rg.2015150118 [Consulta: setiembre 2017].

3. Mettler FA Jr, Bhargavan M, Faulkner K, Gilley DB, Gray JE, Ibbott GS, et al. Radiologic and nuclear medicine studies in the United States and worldwide: frequency, radiation dose, and comparison with other radiation sources-1950-2007. Radiology 2009; 253(2):520-31. Disponible en: http://pubs.rsna.org/doi/10.1148/radiol.2532082010 [Consulta: setiembre 2017].

4. Hendee WR, O'Connor MK. Radiation risks of medical imaging: separating fact from fantasy. Radiology 2012; 264(2):312-21. doi:10.1148/radiol. 12112678

5. Brenner DJ, Hall EJ. Computed tomography - an increasing source of radiation exposure. N Engl J Med 2007; 357(22):2277-84. Disponible en: http://www.nejm.org/doi/ full/10.1056/NEJMra072149 [Consulta: julio 2017].

6. Griffey RT, Sodickson A. Cumulative radiation exposure and cancer risk estimates in emergency department patients undergoing repeat or multiple CT. AJR Am J Roentgenol 2009; 192(4):887-92. doi:10.2214/AJR.08.1351

7. Hricak H, Brenner DJ, Adelstein SJ, Frush DP, Hall EJ, Howell RW, et al. Managing radiation use in medical imaging: a multifaceted challenge. Radiology 2011; 258(3):889-905. Disponible en: http://pubs.rsna.org/doi/ 10.1148/radiol.10101157 [Consulta: setiembre 2017].

8. Shah KH, Slovis BH, Runde D, Godbout B, Newman DH, Lee J. Radiation exposure among patients with the highest CT scan utilization in the emergency department. Emerg Radiol 2013; 20(6):485-91. Disponible en: http://link.sprin- ger.com/10.1007/s10140-013-1142-8 [Consulta: setiembre 2017].

9. Lam DL, Larson DB, Eisenberg JD, Forman HP, Lee CI. Communicating potential radiation-induced cancer risks from medical imaging directly to patients. AJR Am J Roentgenol 2015; 205(5):962-70. Disponible en: http://www.ajronline.org/doi/10.2214/AJR.15.15057 [Consulta: setiembre 2017].

10. Lee CI, Haims AH, Monico EP, Brink JA, Forman HP. Diagnostic CT scans: assessment of patient, physician, and radiologist awareness of radiation dose and possible risks. Radiology 2004; 231(2):393-8. Disponible en: http://pubs.rsna.org/doi/10.1148/radiol.2312030767 [Consulta: setiembre 2017].

11. Ubeda de la Cerda C, Nocetti D, Alarcon R, Insulza A, Calcagno S, Castro M, et al. Magnitudes y unidades para dosimetría a pacientes en radiodiagnóstico e intervencionismo. Rev Chil Radiol 2015; 21(3):94-9. Disponible en: https://scielo.conicyt.cl/pdf/rchradiol/v21n3/art04.pdf [Consulta: setiembre 2017].

12. Andisco D, Blanco S, Buzzi AE. Dosimetría en tomografía computada. Rev Argent Radiol 2014; 78(3):156-60. doi:10.1016/j.rard.2014.07.004

13. Mendizábal Méndez AL. Radiación ionizante en tomografía computada: un tema de reflexión. An Radiol Méx 2012; 2:90-7. Disponible en: http://www.medigraphic.com/ pdfs/anaradmex/arm-2012/arm122d.pdf [Consulta: setiembre 2017].

14. Slovis BH, Shah KH, Yeh DD, Seethala R, Kaafarani HM, Eikermann M, et al. Significant but reasonable radiation exposure from computed tomography-related medical imaging in the ICU. Emerg Radiol 2016; 23(2):141-6. Disponible en: http://link.springer.com/10.1007/s10140-015-1373-y [Consulta: setiembre 2017].

15. Sodickson A, Baeyens PF, Andriole KP, Prevedello LM, Nawfel RD, Hanson R, et al. Recurrent CT, cumulative radiation exposure, and associated radiation-induced cancer risks from CT of adults. Radiology 2009; 251(1):175-84. Disponible en: http://pubs.rsna.org/doi/10.1148/radiol. 2511081296 [Consulta: setiembre 2017].

16. Schonfeld SJ, Lee C, Berrington de González A. Medical exposure to radiation and thyroid cancer. Clin Oncol (R Coll Radiol) 2011; 23(4):244-50. doi:10.1016/j.clon.2011.01.159

17. Comisión Chilena de Energía Nuclear; Vivallo L, Villanueva L, Sanhueza S, comps. Efectos de las radiaciones ionizantes en el ser humano. Santiago: CCHEN, 2010: 38 p. Disponible en: http://oirs.cchen.cl/saber/PDF/efectos_biologicos_mayo2010.pdf [Consulta: setiembre 2017].

18. Smith G. UNSCEAR 2013 Report. Vol I: Report to the General Assembly, Annex A: Levels and effects of radiation exposure due to the nuclear accident after the 2011 great east-Japan earthquake and tsunami. United Nations Scientific Committee on the Effects of Atomic Radiation New York: United Nations (2014). [Book Review) J Radiol Prot 2014; 34:725-7. Disponible en: http://iopscience.iop.org/arti- 
cle/10.1088/0952-4746/34/3/B01/pdf [Consulta: setiembre 2017].

19. Ozasa K, Shimizu Y, Suyama A, Kasagi F, Soda M, Grant EJ, et al. Studies of the mortality of atomic bomb survivors, Report 14, 1950-2003: an overview of cancer and noncancer diseases. Radiat Res 2012; 177(3):229-43. Disponible en: http://www.bioone.org/doi/10.1667/RR2629.1 [Consulta: setiembre 2017].

20. Pierce DA, Preston DL. Radiation-related cancer risks at low doses among atomic bomb survivors. Radiat Res 2000; 154(2):178-86. doi: 10.1667/0033-7587(2000)154 [0178:RRCRAL]2.0.CO;2

21. Preston DL, Shimizu Y, Pierce DA, Suyama A, Mabuchi K. Studies of mortality of atomic bomb survivors. Report 13: solid cancer and noncancer disease mortality: 1950-1997. Radiat Res 2003; 160(4):381-407. Disponible en: http://www.bioone.org/doi/10.1667/0033-7587\% 282000\%29154\%5B0178\%3ARRCRAL\%5D2.0.CO\%3B2 [Consulta: setiembre 2017].

22. Shimizu Y, Pierce DA, Preston DL, Mabuchi K. Studies of the mortality of atomic bomb survivors. Report 12, part II. Noncancer mortality: 1950-1990. Radiat Res 1999; 152(4):374-89. Disponible en: http://www.rrjournal.org/ doi/pdf/10.2307/3580222 [Consulta: setiembre 2017].

23. Pierce DA, Shimizu Y, Preston DL, Vaeth M, Mabuchi K. Studies of the mortality of atomic bomb survivors. Report 12, Part I. Cancer: 1950-1990. Radiat Res 2012; 178(2):AV61-AV87. Disponible en: http://www.bioone.org/doi/10.1667/RRAV06.1 [Consulta: setiembre 2017].

24. Pearce MS, Salotti JA, Little MP, McHugh K, Lee C, Kim $\mathbf{K P}$, et al. Radiation exposure from CT scans in childhood and subsequent risk of leukaemia and brain tumours: a retrospective cohort study. Lancet 2012; 380(9840):499-505. Disponible en: http://www.thelancet.com/journals/lancet/arti-
cle/PIIS0140-6736(12)60815-0/fulltext [Consulta: setiembre 2017].

25. National Research Council. Health risks from exposure to low levels of ionizing radiation: BEIR VII Phase 2. Washington, DC: The National Academies, 2006. doi:10.17226/11340

26. Huda W, Ogden KM, Khorasani MR. Converting dose-length product to effective dose at CT. Radiology 2008; 248(3):995-1003. doi:10.1148/radiol.2483071964

27. Uruguay. UdelaR, Facultad de Medicina. Hospital de Clínicas, Dr. Manuel Quintela. Indicadores hospitalarios. Disponible en: http://www.hc.edu.uy/index.php/conozca-el-hc/indicadores [Consulta: octubre 2017].

28. Brenner DJ, Doll R, Goodhead DT, Hall EJ, Land CE, Little JB, et al. Cancer risks attributable to low doses of ionizing radiation: assessing what we really know. Proc Natl Acad Sci USA 2003; 100(24):13761-6. Disponible en: https:/www.ncbi.nlm.nih.gov/pmc/articles/PMC283495/ [Consulta: octubre 2017].

29. Einstein AJ. Medical imaging: the radiation issue. Nat Rev Cardiol 2009; 6(6):436-8. Disponible en: https://www. ncbi.nlm.nih.gov/pmc/articles/PMC3684954/ [Consulta: octubre 2017].

30. Sociedad Argentina de Radiología, coord. Guía de recomendaciones para la correcta solicitud de pruebas de Diagnóstico por Imagen. Disponible en: http://www. aac.org.ar/imagenes/guias/guia_solic_diag_x_imagenes.pdf [Consulta: octubre 2017].

31. Kanal KM, Butler PF, Sengupta D, Bhargavan-Chatfield M, Coombs LP, Morin RL. U.S. diagnostic reference levels and achievable doses for 10 adult CT examinations. Radiology 2017; 284(1):120-33. doi:10.1148/radiol.2017161911

32. Directives: Council Directive 2013/59/EURATOM of 5 december 2013. OJ 2014; L13(57):1-73. 